\title{
Advances in chemical investigations of the heaviest elements
}

\author{
Andreas Türler ${ }^{1,2,3}$ \\ ${ }^{1}$ Paul Scherrer Institut, Department Nuclear Energy and Safety, 5232 Villigen PSI, Switzerland \\ ${ }^{2}$ University of Bern, Department of Chemistry and Biochemistry, 3012 Bern, Switzerland \\ ${ }^{3}$ University of Bern, Albert Einstein Center for Fundamental Physics, 3012 Bern, Switzerland
}

\begin{abstract}
Although somewhat in the shadow of the discoveries of new elements, experimental chemical investigations of the heaviest elements have made tremendous progress in the last decades. Indeed, it was possible to experimentally determine thermochemical properties of heavy transactinide elements such as copernicium or flerovium. But will it be possible to chemically study all currently known elements of the periodic table up to element 118 ? While it is experimentally feasible to work with single atoms, the short half-lives of even the longest currently known isotopes of elements 115 through 118 call for new experimental approaches.
\end{abstract}

\section{Introduction}

Recently, the discovery of elements with atomic numbers 113,115, 117, and 118 has been validated by a joint IUPAC and IUPAP working group and attribution of priority of discovery to a laboratory or to a collaborating group of laboratories has been made [1,2]. It should be noted that these findings were not in all parts unanimously welcomed by the scientific community. The reservations concern the hitherto proposed cross-reaction link between alpha-decay chains associated with the isotopes ${ }^{293} 117$ and ${ }^{289} 115$, which was shown to not withstand a rigorous statistical treatment $[3,4]$. Nevertheless, the respective discoverers were invited to propose a name and a symbol to the IUPAC Inorganic Chemistry in accordance with IUPAC recommendations on how to name a new element, which was recently revised [5]. The most important adjustment was that the names of new elements should have an ending that "reflects and maintains historical and chemical consistency. This would be in general "-ium" for elements belonging to groups 1-16, i.e. including the f-block elements, "-ine" for elements of group 17 and "-on" for elements of group 18." The positioning of a newly discovered element in a group of the periodic table is not considered or discussed in the published discovery profiles $[1,2]$ and IUPAC proceeded to place elements with atomic numbers $113,115,117$ and 118 into groups 13, 15, 17, and 18 respectively, without offering any further considerations. But interestingly, IUPAC has no recommendation for a specific form of the periodic table. Indeed, an IUPAC project has been recently initiated [6] to resolve the question whether group 3 consists of the elements Sc, Y, Lu, and Lr or, of the elements Sc, Y, La and Ac? This dispute has no influence on the name of the involved elements; however, the misplacement of an element in groups 17 and 18 would definitely affect their suffixes. Currently, the discovery profiles of the four new elements concentrate mainly on the nuclear aspects in order to prove that indeed a new element with a previously unreported atomic number was synthesized. The place an element occupies in the Periodic Table is not only defined by its atomic number, i.e. the number of protons in the nucleus, but also by 
its electronic configuration, which defines its chemical properties. Strictly speaking, a new element is assigned its proper place only after its chemical properties have been sufficiently investigated. No chemical information was obtained whatsoever. Is it then conceivable, that the shape of the periodic table may be altered in the future and newly discovered elements are moved away from their current position to different groups? Not very likely for elements with atomic numbers $113,115,117$, and 118 , as recent theoretical calculations have shown (see Ref. [7] for a review). Nevertheless, strong relativistic effects significantly influence the chemical behaviour of the heaviest elements and, therefore, experimental chemical investigations of these elements are warranted and of decisive importance.

The study of the chemical properties of the heaviest known elements in the Periodic Table is an extremely challenging task and requires the development of unique methods, but also the persistence to continuously improve all the processes and components involved in order to achieve the ultimate goal of chemically isolating one single atom that lives for only a few seconds (or even less). Due to the low production rates of the heaviest elements, chemical information obtained from experiments is limited to the knowledge of very few basic properties. It mainly answers the question whether a new element behaves similarly to its lighter congeners in a chemical group, or whether some deviations from the trends occur due to very strong relativistic effects on its valence electron shells. Indeed, in some cases it has been possible to experimentally investigate chemical properties of transactinide elements and even synthesize simple compounds. The half-life limitation of current chemical systems allow only investigations of transactinide nuclides with $T_{1 / 2}>1 \mathrm{~s}$. Thus the heaviest element whose chemical properties have been investigated experimentally is flerovium $(Z=114, \mathrm{Fl})$ $[8,9]$. While the body of experiments and data on the chemistry of transactinide elements is already impressive, pushing the limits to investigations of even heavier and shorter-lived elements will require the improvement of existing technologies and the development of new techniques. For the not yet studied elements, like Mt, Ds, and Rg, isotopes with $T_{1 / 2}$ suitable for chemical studies have already been identified. Currently, also element 115 may come within reach of present day technologies.

\section{Basic considerations and requirements}

A chemistry experiment with a transactinide element can be divided into three basic steps: first, synthesis of the transactinide nuclide, second, rapid transport of the synthesized nuclide to the chemical apparatus, third, fast chemical isolation of the desired nuclide, preparation of a sample suitable for nuclear spectroscopy, and detection of the nuclide via its characteristic nuclear decay properties.

The synthesis of suitable, sufficiently long-lived transactinide nuclei is accomplished in the same manner as in the nuclear physics discovery experiments. High intensity heavyion beams such as ${ }^{18} \mathrm{O},{ }^{22} \mathrm{Ne},{ }^{26} \mathrm{Mg},{ }^{36} \mathrm{~S}$, and mainly ${ }^{48} \mathrm{Ca}$ are directed at exotic, highly radioactive target nuclides such as ${ }^{244} \mathrm{Pu},{ }^{243} \mathrm{Am},{ }^{248} \mathrm{Cm},{ }^{249} \mathrm{Bk}$, or ${ }^{249,251} \mathrm{Cf}$. On the one hand as intense beams as possible are to be used; on the other hand the destruction of the very valuable and highly radioactive targets has to be avoided. Production rates are proportional to both the target thickness and the beam intensity. For nuclear reactions involving the lighter projectiles, target thickness is limiting the compound nucleus recoil ranges $\left(<1 \mathrm{mg} / \mathrm{cm}^{2}\right)$, because the separation techniques used require the compound nucleus products to recoil out of the target. With the higher- $Z$ projectiles, the recoil ranges are larger, but because of the higher rate of projectile energy loss in the target material and the narrow projectile energy range effective for heavy-element production, the effective target thicknesses are once again limited to approximately $1 \mathrm{mg} / \mathrm{cm}^{2}$. 

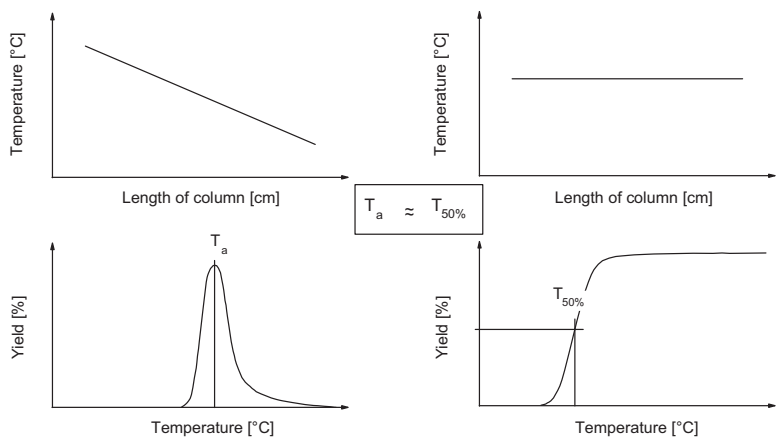

Figure 1. Upper panel: temperature profiles employed in TC and IC; lower panel: deposition peak and integral chromatogram resulting from TC and IC, respectively.

Since transactinide nuclei are inevitably produced among many orders of magnitude larger amounts of "background" activities, which interfere with the detection and identification of the decay of transactinide atoms, there is nowadays a recognized need for physical pre-separation of the transactinide atoms before chemical investigation. Except for the most asymmetric synthesis reactions, the recoil energy of the fusion reaction products imparted by the momentum of the beam is sufficient to allow the separated nuclei to pass through a thin Mylar ${ }^{\circledR}$ window, which separates the low pressure in the separator (i.e. 1 mbar or vacuum) from the stopping gas cell (i.e. 1 bar). The thin windows are supported by honeycomb grids. Thus, a recoil chamber can be coupled to a kinematic pre-separator instead of a focal plane detector array, making transactinide atoms available for chemical experiments. Since the separated transactinides are usually thermalized in a gas, rapid gasphase chemical reactions and separations are the method of choice. Gas-phase separation procedures are fast, efficient and can be performed continuously, which is highly desirable in order to achieve high overall yields. Two different types of gas chromatographic separations have been developed for the experimental investigation of volatile transactinide elements or compounds, isothermal chromatography (IC) and thermochromatography (TC). A schematic of the operating principle of IC and TC is provided in Fig. 1.

In IC, a carrier gas is flowing through a chromatography column of constant, isothermal temperature. Depending on the temperature and on the enthalpy of adsorption, $\Delta H_{a d s}$, of the species on the column surface, the species travel slower through the length of the column than the carrier gas. This retention time can be determined by continuously introducing a shortlived nuclide into the column and detecting the fraction of nuclides that have decayed at the exit of the column. A characteristic quantity is the temperature at which half of the introduced nuclides are detected at the exit $\left(T_{50 \%}\right)$. In this case, the retention time in the column is equal to $t_{1 / 2}$ of the introduced nuclide. Since volatile species rapidly emerge at the exit of the column, they can be condensed and assayed with nuclear spectroscopic methods. This is done by turbulent mixing of the gas stream exiting the chromatography column (containing the volatile species under investigation) with a second gas stream containing aerosol particles. Volatile species will condense on the surface of these aerosol particles and can be transported to a counting device, i.e. a rotating wheel system or a tape system, where they are collected by impaction and subsequently assayed by $\alpha$-particle and SF-spectroscopy.

In TC, a carrier gas is flowing through a chromatography column, to which a negative longitudinal temperature gradient has been applied. Species that are volatile at the starting 
point are transported downstream of the column by the carrier gas flow. Due to the decreasing temperature in the column, the time the species spend in the adsorbed state increases exponentially. Different species form distinct deposition peaks, depending on their $\Delta H_{a d s}$ on the column surface and are thus separated from each other. A characteristic quantity is the deposition temperature $\left(T_{a}\right)$, which depends on various experimental parameters. The mixture of species to be separated can be injected continuously. The deposition position (and thus $T_{a}$ ) of each single radioactive decay in the column contributes chemical information about $\Delta H_{a d s}$ of the investigated species. In recent years, TC detectors proved enormously successful. The carrier gas containing volatile atoms or molecules is flowing through a narrow channel formed by a series of planar silicon diodes. Due to the close proximity of the silicon diodes facing each other, the probability to register a complete decay chain consisting of a series of $\alpha$-particle decays is very high. Serious limitations constitute the required operating conditions of silicon diodes, which sustain only temperatures from $+35^{\circ} \mathrm{C}$ to about $-180^{\circ} \mathrm{C}$.

A new development concerns vacuum chromatography, where, under molecular flow conditions, atoms or molecules can, in principle, be isolated within about 100 milliseconds. In conjunction with diamond detectors that can be operated up to $180^{\circ} \mathrm{C}$, so far inaccessible elements beyond $\mathrm{Fl}$ can be accessed in the future. These developments will be presented later in the form of a proof-of-principle experiment with relatively short-lived $\mathrm{Tl}$ nuclides as homologs of element 113.

\section{Recent accomplishments and selected results}

With the introduction of silicon based cryo-thermochromatography detectors a number of breakthroughs were made possible. This was mainly due to the much improved overall efficiency of the chemical systems provided by these detectors. While the overall efficiency to detect a three member decay chain in IC-experiments with $\mathrm{Bh}(Z=107)$ [10] was estimated at a meager $4 \%$, this number increased by more than one order of magnitude to about $50 \%$ in experiments with Hs $(Z=108)$ [11]. This accomplishment also allowed for the discovery of two new Hs isotopes, ${ }^{270} \mathrm{Hs}$ and ${ }^{271} \mathrm{Hs}$, and their hitherto unknown daughter nuclei, ${ }^{266} \mathrm{Sg}$ and ${ }^{267} \mathrm{Sg}$ and ${ }^{263} \mathrm{Rf}$, respectively. The success of these experiments paved the way to first ever chemical investigations of copernicium $(Z=112, \mathrm{Cn})[12]$ and $\mathrm{Fl}(Z=114)[8,9]$ in the elemental state, but also to the first characterization of a volatile $\operatorname{Sg}(\mathrm{CO})_{6}$ compound [13]. The results of these experiments have been reviewed already extensively [14] and will also be the topic of several contributions to the current conference proceedings.

An interesting development concerns investigations of the interaction of superheavy elements $\mathrm{Cn}$ and Fl with Se surfaces. The stability of Fl-selenides is expected to be the highest in group 14, whereas it is the lowest for Cn-selenides in group 12. Both selenides are expected to be the most volatile within their respective groups. First experiments revealed surprising results for the behavior of $\mathrm{Cn}$ versus its lighter homolog $\mathrm{Hg}$. Further experiments are in progress to elucidate the behavior of $\mathrm{Cn}$ - and $\mathrm{Fl}$-selenides.

The recent introduction of carbonyl complex chemistry to the heavy element region opens up new possibilities to synthesize and study other transactinide containing carbonyl compounds with the elements Bh, Hs, and Mt. Furthermore, the thermal stability towards decomposition of $\mathrm{Sg}(\mathrm{CO})_{6}$ was studied in a recent experiment by an international collaboration at RIKEN, Japan. The data of these experiments is still under analysis.

\section{Vacuum chromatography with heavy transactinide elements}

Based on trends of the standard enthalpies of monoatomic gaseous elements in the periodic table, all transactinide elements in groups 12 through 17 and groups 1 and 2 are expected 


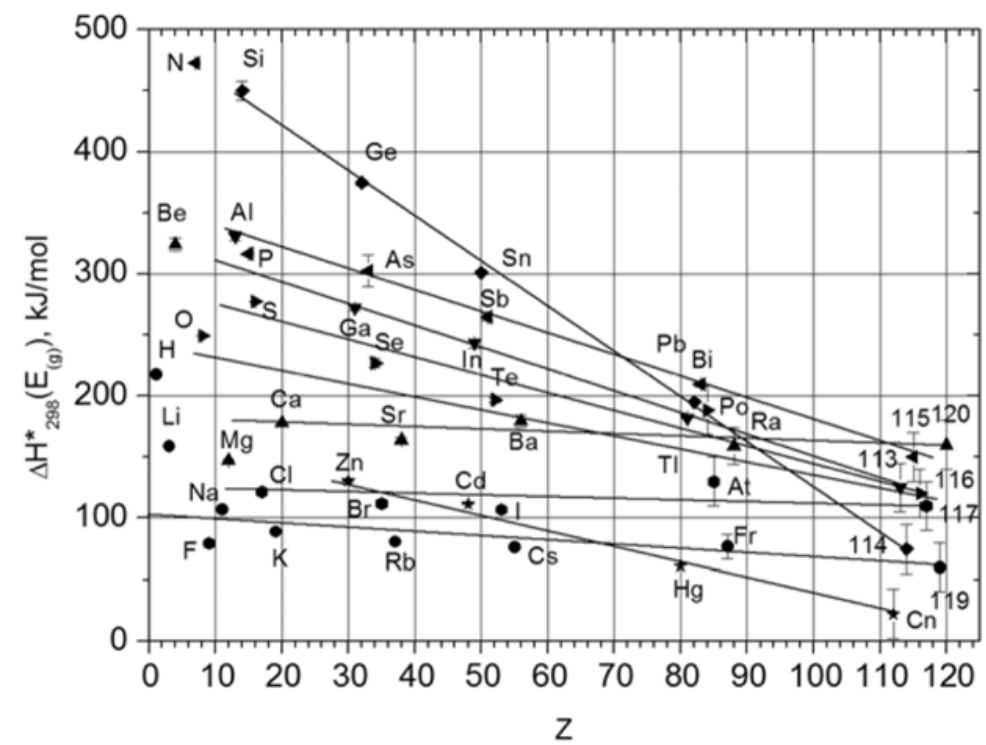

Figure 2. Extrapolation of the standard enthalpies of monoatomic gaseous elements $\Delta H^{*}{ }_{298}\left(\mathrm{E}_{(g)}\right)$ along the groups of the Periodic Table based on the atomic number $Z$ (adapted from [15]).

to be the most volatile ones in the group. A simple and very early extrapolation of the standard enthalpies of monoatomic gaseous elements, $\Delta H^{*}{ }_{298}\left(\mathrm{E}_{(\mathrm{g})}\right)$ over the atomic number $Z$, predicts the heavy transactinides $\mathrm{Cn}$ through element 120 [15] to be rather volatile (Fig. 2), since the standard enthalpies of monoatomic gases are mostly equal to the standard sublimation enthalpy $\Delta H_{S}$ of these elements. Those linear extrapolations should be used with care, as there is no solid theoretical basis for them. However, the high volatility of $\mathrm{Cn}$ and Fl has been demonstrated experimentally and was predicted by much more sophisticated relativistic theoretical calculations. One might therefore argue that elements such as $\mathrm{Tl}$ or At serve as suitable homologs for the development of new chemistry setups for investigations of elements 113, 115, Lv, and 117. Depending on the $\Delta H_{S}$ of these elements, column temperatures of up to about $700{ }^{\circ} \mathrm{C}$ will be required to rapidly transmit them through an open chromatography column at vacuum conditions.

A suitable isothermal vacuum chromatography (IVAC) setup was constructed at Paul Scherrer Institute, Switzerland and used to investigate the volatility of $\mathrm{Tl}$ on $\mathrm{SiO}_{2}$ at the single-atom level in an experiment at JAEA, Japan [16]. A mass separated low-energy ion beam of ${ }^{184} \mathrm{Tl}^{+}$ions was provided by the JAEA-ISOL system at the tandem accelerator. A 3D construction drawing of the IVAC system is provided in Fig. 3. The low energy ${ }^{184} \mathrm{Tl}^{+}$beam enters the Hf hot catcher (HC) through a small hole. Neutralized ${ }^{184} \mathrm{Tl}$ atoms exit the hot catcher and enter the kinked quartz chromatography column in the isothermal oven. The kink prevents $\mathrm{Tl}$ atoms from traversing the chromatography column in a straight flight path without surface interactions. At the exit of the column a four-fold diamond detector is registering $\alpha$-particle decays of condensed ${ }^{184} \mathrm{Tl}$. As the transport of the released atoms is governed exclusively by random walk, the number of surface interactions is directly proportional to the geometric area. This means that the probability for an atom to leave the HC containment through the 6-mm exit hole into the chromatography column is four times greater (80\%) than for it to exit back through the 3-mm entrance orifice (20\%). However, as the conductance of the chromatography column is rather low, the bulk of the species under observation re-enters 


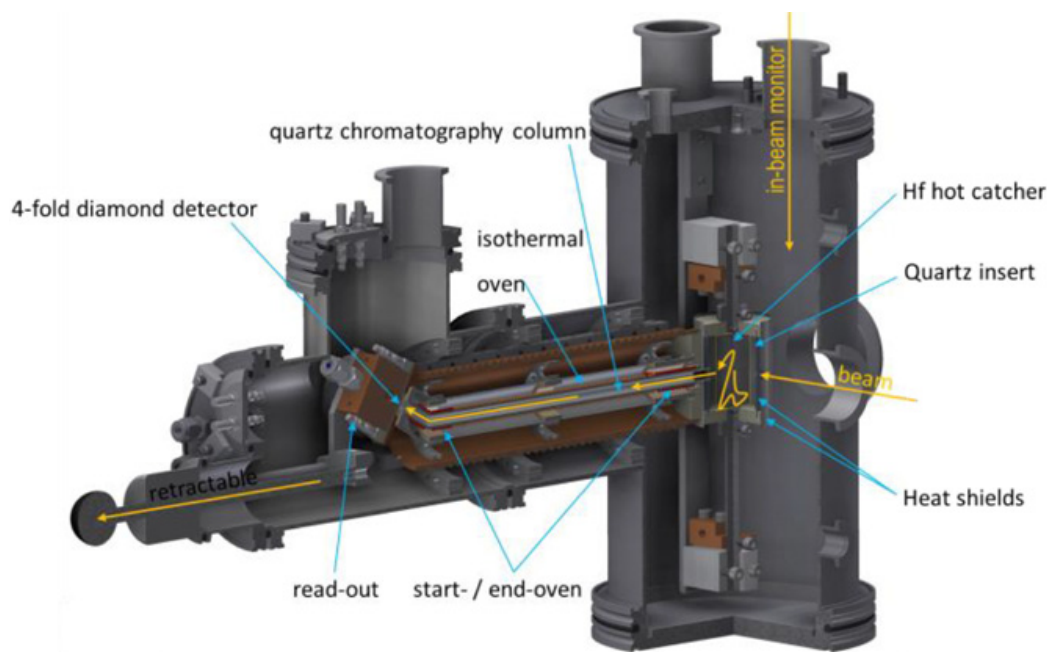

Figure 3. Construction drawing of the IVAC setup.

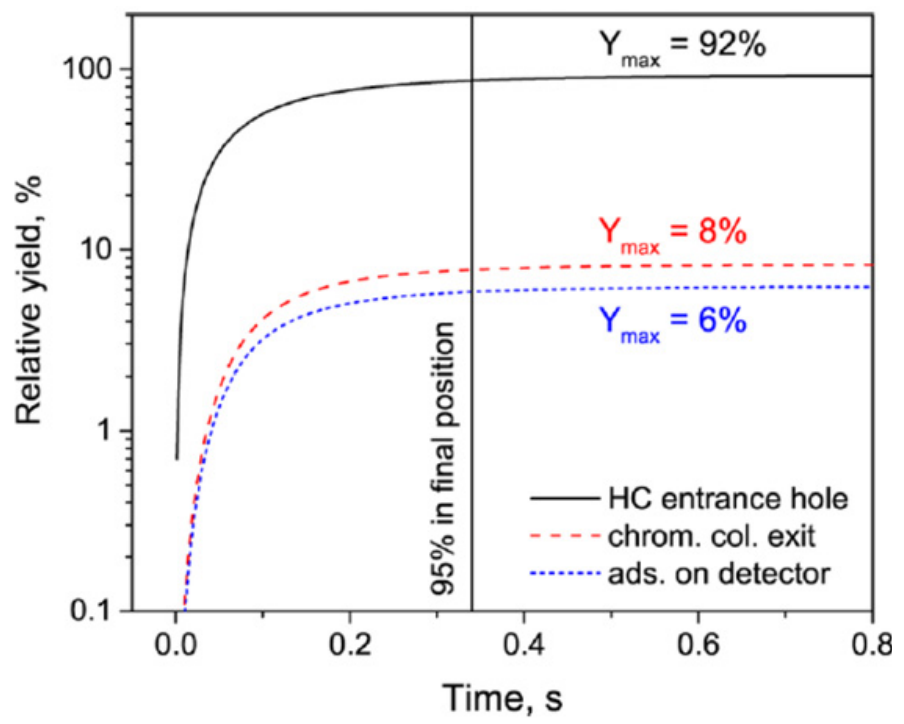

Figure 4. Simulated transmission efficiency through the IVAC setup assuming a temperature independent adsorption behavior as a function of time. Figure from [16].

the HC containment multiple times and eventually leaves it through the small entrance orifice, leading to its ultimate loss.

In Fig. 4 the transmission efficiency through the setup simulated with the COMSOL multiphysics mathematical particle tracing module is shown (assuming no temperature dependent retention). A major portion (92\%) of all inserted $\mathrm{Tl}$ atoms escape through the HC entrance orifice (black solid line), whereas $8 \%$ (red dashed line) reach the end of the chromatography column, and $6 \%$ (blue dotted line) adsorb on the active area of the detector. 


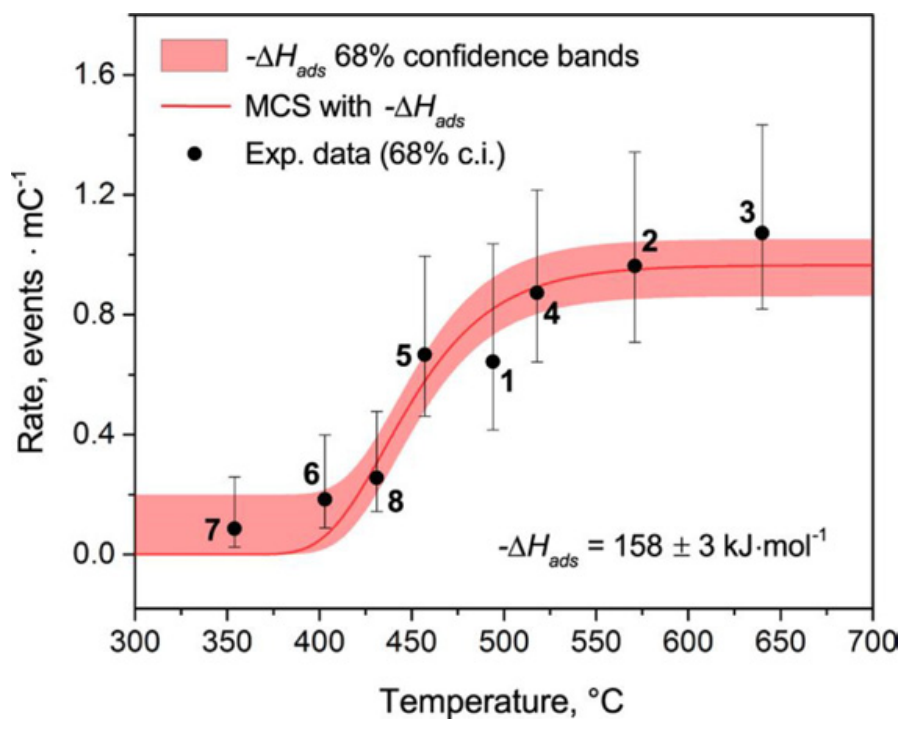

Figure 5. Measured event rate of ${ }^{184} \mathrm{Tl}$ decays in the diamond detector at the exit of the column as a function of isothermal temperature. For a detailed description see text. Figure from [16].

After $100 \mathrm{~ms} 50 \%$ of all atoms that will reach the column exit have already reached it. The vertical line at $340 \mathrm{~ms}$ indicates the time at which $95 \%$ of all atoms are in their final position. The measured transmission efficiency between the in-beam monitor and the exit detector amounted to only $2.2 \%$ (compared to a theoretical yield of $6 \%$ ). This low yield would clearly be insufficient for an experiment with transactinide elements. A mechanism has to be developed to transmit the product beam through a thin hot window into the HC. This way the huge losses through the entrance orifice (92\%) could be avoided and the yield would be increased significantly. This should be feasible as the product beam from a gasfilled separator or a velocity filter has a much higher energy $(20-50 \mathrm{MeV})$ compared to the one from the JAEA-ISOL system.

In Fig. 5, the experimentally measured external chromatogram (black dots, error bars: $68 \%$ c.i.) for $\mathrm{Tl}$ atoms passing through a quartz column of the IVAC setup is shown. The data was modelled using a Monte Carlo simulation procedure assuming an adsorption enthalpy of $-\Delta H_{\mathrm{ads}}^{\mathrm{SiO}_{2}}(\mathrm{Tl})=(158 \pm 3) \mathrm{kJ} \cdot \mathrm{mol}^{-1}$ (red line) and the corresponding confidence bands (red area); the bold black numbers indicate the sequence of the conducted measurements.

The determined adsorption enthalpy differed noticeably from previously reported values of $-\Delta H_{a d s}=137 \mathrm{~kJ} \cdot \mathrm{mol}^{-1}$ that could eventually be attributed to the formation of TlOH. In this experiment, due to vacuum conditions, there were negligible particle-particle interactions in the molecular flow regime. Furthermore, high temperatures of $\approx 1000^{\circ} \mathrm{C}$ were applied all along the quartz chromatography column prior to the experiment, so that reactions with $\mathrm{O}_{2}$ and $\mathrm{H}_{2} \mathrm{O}$ were virtually excluded. A further indication that indeed the adsorption of elemental $\mathrm{Tl}$ on $\mathrm{SiO}_{2}$ was investigated was that the newly determined adsorption enthalpy value now perfectly fell in line with the empirical correlation function between the sublimation enthalpies of various elements and their respective adsorption enthalpies on a quartz surface [16], which can be described as:

$$
-\Delta H_{\mathrm{ads}}^{\mathrm{SiO}_{2}}=(0.84 \pm 0.07) \cdot \Delta H_{\mathrm{subl}}^{\circ}-(2.8 \pm 8.9) \mathrm{kJ} \cdot \mathrm{mol}^{-1}
$$




\section{Conclusions}

Applying vacuum chromatography in conjunction with diamond detectors may open up new avenues of transactinide research and will allow chemical studies of nuclides with half-lives as short as $100 \mathrm{~ms}$ or even less. However, the modest yields of first "proof-ofprinciple" experiments clearly demonstrate the need for further significant improvements, before experiments with trans-flerovium elements can be attempted with a realistic chance of success. While looking at possible chemical experiments with the heaviest known elements, one should not forget about the fact, that so far no chemical studies of elements Mt, Ds, and $\mathrm{Rg}$ have been conducted, even though isotopes with half-lives $>1 \mathrm{~s}$ are already known. Also, results on the chemical properties of element 113 are not convincing so far $[14,17]$.

\section{References}

[1] P.J. Karol et al., Pure Appl. Chem. 88, 139 (2016).

[2] P.J. Karol et al., Pure Appl. Chem. 88, 155 (2016).

[3] U. Forsberg et al., Nucl. Phys. A953, 117 (2016).

[4] U. Forsberg et al., Phys. Lett. B760, 293 (2016).

[5] W.H. Koppenol et al., Pure Appl. Chem. 88, 401 (2016).

[6] E. Scerri et al., Chemistry International 38, 22 (2016); see also: www.iupac.org/ project/2015-039-2-200.

[7] A. Türler and V. Pershina, Chem. Rev. 113, 1237 (2013).

[8] R. Eichler et al., Radiochim. Acta 98, 133 (2010).

[9] A. Yakushev et al., Inorg. Chem. 53, 1624 (2014).

[10] R. Eichler et al., Nature 407, 63 (2000).

[11] Ch.E. Düllmann et al., Nature 418, 859 (2002).

[12] R. Eichler et al., Nature 447, 72 (2007).

[13] J. Even et al., Science 345, 1491 (2014).

[14] A. Türler, R. Eichler, A. Yakushev, Nucl. Phys. A944, 640 (2015).

[15] B. Eichler, Kernenergie 19, 307 (1976).

[16] P. Steinegger et al., J. Phys. Chem. C 120, 7122 (2016).

[17] S.N. Dmitriev et al., Mendeleev Commun. 24, 253 (2014). 